\title{
On invariants for the Poincaré equations and applications
}

\author{
Valeriy Imaykin \\ Diagnostic Equipment of Non-Destructive Control, LLC \\ Proezd Olminskogo 3A, Moscow, 129164 Russia \\ email: ivm61@mail.ru
}

Alexander Komech

Faculty of Mathematics of Vienna University, Oskar-Morgenstern-Platz 1, 1090 Vienna, Austria and IITP RAS, Bolshoy Karetny per. 19-1, 127051 Moscow, Russia

email: alexander.komech@univie.ac.at

Herbert Spohn

Zentrum Mathematik, TU München

Boltzmannstr. 3, Garching, 85747 Germany

email: spohn@ma.tum.de

\begin{abstract}
We extend the Noether theory of invariants to the Poincaré equations. We apply this extension to the Maxwell-Lorentz equations coupled to the Abraham rotating extended electron with the configuration space $S O(3)$.

Keywords: Poincaré equations; conservation laws; Noether theory of invariants; Abraham's rotating extended electron; Maxwell-Lorentz equations; Hamilton's least action principle.
\end{abstract}




\section{Introduction}

For the Maxwell-Lorentz equations with a rotating charged particle (see Eqs. (2.3)-(2.6) below) Hamilton's least action principle is justified in [3]. The main contribution of [3] is variational derivation of the Lorentz torque equation (2.6). While the equations (2.3)-(2.5) follow by standard Euler-Lagrange arguments, the Lorentz torque equation (2.6) follows by variational Poincaré equations [2, 6].

Our main result is a suitable generalization of the Noether theory of invariants to the Poincaré equations on the Lie groups. Moreover, we apply this generalization to a formal derivation of conservation laws for the Maxwell-Lorentz equations with a rotating charged particle. We show that the corresponding "Poincaré invariants" coincide with classical known expressions considered in [4] where their conservation was shown by direct calculation.

We consider solutions for which all our formal differentiations and integration by parts hold true.

\section{Maxwell-Lorentz equations}

The Maxwell fields $E(x, t)$ and $B(x, t)$ are generated by motion of a rotating charge. External fields $E^{e x t}$ and $B^{e x t}$ are generated by the corresponding external charges and currents. Let the rotating charge be centered at the position $q$ with the velocity $\dot{q}$. For simplicity we assume that the mass distribution, $m \rho(x)$, and the charge distribution, e $\rho(x)$, are proportional to each other. Here $m$ is the total mass, $e$ is the total charge, and we use a system of units such that $m=1$ and $e=1$. The coupling function $\rho(x)$ is a sufficiently smooth radially symmetric function of fast decay as $|x| \rightarrow \infty$,

$$
\rho(x)=\rho_{r}(|x|)
$$

\subsection{Angular velocity}

Let us denote by $\omega(t) \in \mathbb{R}^{3}$ the angular velocity "in space" (in the terminology of [2]) of the charge. Namely, let us fix a "center" point $O$ of the rigid body. Then the trajectory of each fixed point of the body is described by

$$
x(t)=q(t)+R(t)(x(0)-q(0))
$$

where $q(t)$ is the position of $O$ at the time $t$, and $R(t) \in S O(3)$. Respectively, the velocity reads

$$
\dot{x}(t)=\dot{q}(t)+\dot{R}(t)(x(0)-q(0))=\dot{q}(t)+\dot{R}(t) R^{-1}(t)(x(t)-q(t))=\dot{q}(t)+\omega(t) \wedge(x(t)-q(t)),
$$

where $\omega(t) \in \mathbb{R}^{3}$ corresponds to the skew-symmetric matrix $\dot{R}(t) R^{-1}(t)$ by the rule

$$
\dot{R}(t) R^{-1}(t)=\mathcal{J} \omega(t):=\left(\begin{array}{ccc}
0 & -\omega_{3}(t) & \omega_{2}(t) \\
\omega_{3}(t) & 0 & -\omega_{1}(t) \\
-\omega_{2}(t) & \omega_{1}(t) & 0
\end{array}\right) .
$$

We assume that $x$ and $q$ refer to a certain Euclidean coordinate system in $\mathbb{R}^{3}$, and the vector product $\wedge$ is defined in this system by standard formulas. The identification (2.2) of a skew-symmetric matrix and the corresponding angular velocity vector is true in any Euclidean coordinate system of the same orientation as the initial one. 


\subsection{Dynamical equations}

Then the system of Maxwell-Lorentz equations with spin reads, see [4, 7]

$$
\begin{array}{cc}
\dot{E}=\nabla \wedge B-(\dot{q}+\omega \wedge(x-q)) \rho(x-q) & (a), \quad \dot{B}=-\nabla \wedge E \quad(b), \\
\nabla \cdot E(x, t)=\rho(x-q(t)) & (a), \quad \nabla \cdot B(x, t)=0 \quad(b), \\
\ddot{q}=\int\left[E+E^{e x t}+(\dot{q}+\omega \wedge(x-q)) \wedge\left(B+B^{e x t}\right)\right] \rho(x-q) d x \\
I \dot{\omega}=\int(x-q) \wedge\left[E+E^{e x t}+(\dot{q}+\omega \wedge(x-q)) \wedge\left(B+B^{e x t}\right)\right] \rho(x-q) d x,
\end{array}
$$

where $I$ is the moment of inertia defined by

$$
I=\frac{2}{3} \int x^{2} \rho(x) d x .
$$

Here the equations (2.3) are Maxwell equations with the corresponding charge density and current, equations (2.4) are constraints. The back reaction of the field onto the particle is given through the Lorentz force equation (2.5), and the Lorentz torque equation (2.6) deals with rotational degrees of freedom.

\subsection{The variational Hamilton principle}

Let us introduce electromagnetic potentials $\mathcal{A}=\left(A_{0}, A\right), \mathcal{A}^{\text {ext }}=\left(A_{0}^{\text {ext }}, A^{\text {ext }}\right)$ :

$$
\begin{gathered}
B=\nabla \wedge A, \quad E=-\nabla A_{0}-\dot{A} . \\
B^{e x t}=\nabla \wedge A^{e x t}, \quad E^{e x t}=-\nabla A_{0}^{e x t}-\dot{A}^{e x t} .
\end{gathered}
$$

Next we define the Lagrangian

$$
\begin{aligned}
& L(\mathcal{A}, q, R, \dot{\mathcal{A}}, \dot{q}, \dot{R})=\frac{1}{2} \int\left(E^{2}(x)-B^{2}(x)\right) d x+\frac{1}{2} \dot{q}^{2}+\frac{1}{2} I \omega^{2} \\
& -\int\left[A_{0}(x)+A_{0}^{e x t}(x)\right] \rho(x-q) d x+\int(\dot{q}+\omega \wedge(x-q)) \cdot\left[A(x)+A^{e x t}(x)\right] \rho(x-q) d x,
\end{aligned}
$$

where $E(x)$ and $B(x)$ are expressed in terms of $\mathcal{A}(x)$ and $\dot{\mathcal{A}}(x)$ according to (2.8), and $\omega=$ $\mathcal{J}^{-1} \dot{R} R^{-1}$ by (2.2).

This Lagrangian functional depends on $R$ only trough $\omega$ due to the spherical symmetry of the charge and mass distributions (C). Respectively, the dynamical equations (2.3) $-(2.6)$ involve $R$ only through $\omega$ as well. On the other hand, in the case of non-radial densities the Lagrangian and the equations involve $R$ explicitly, and the moment of inertia $I$ becomes a matrix with $x \otimes x$ instead of $x^{2}$ in (2.7).

The corresponding action functional has the form

$$
S=S(\mathcal{A}, q, R):=\int_{t_{1}}^{t_{2}} L(\mathcal{A}(t), q(t), R(t), \dot{\mathcal{A}}(t), \dot{q}(t), \dot{R}(t)) d t
$$

Then the Hamilton's least action principle reads

$$
\delta S(\mathcal{A}, q, R)=0,
$$


where the variation is taken over $\mathcal{A}(t), q(t), R(t)$ with the boundary conditions

$$
\left.(\delta \mathcal{A}, \delta q, \delta R)\right|_{t=t_{1}}=\left.(\delta \mathcal{A}, \delta q, \delta R)\right|_{t=t_{2}}=0 .
$$

Regular solutions and external potential. Everywhere below we consider regular solutions to the system (2.3) -(2.6). This means that $q \in C^{2}\left(\mathbb{R}, \mathbb{R}^{3}\right), \omega \in C^{1}\left(\mathbb{R}, \mathbb{R}^{3}\right)$, and all the involved functions and fields/potentials are sufficiently smooth and have (with all the necessary derivatives) a sufficient decay as $|x| \rightarrow \infty$ so that the partial integrations below are allowed.

In [3, Theorem 2.1] we have shown that for regular solutions, the Maxwell-Lorentz system (2.3) $-(2.6)$ is equivalent to the least action principle (2.12) $-(2.13)$ In detail, consider the variational equations

$$
\frac{\delta S}{\delta \mathcal{A}}=0 \quad(a), \quad \frac{\delta S}{\delta q}=0 \quad(b), \quad \frac{\delta S}{\delta R}=0 \quad(c) .
$$

Then (2.14), (a), (b) are equivalent respectively to the standard Euler-Lagrange equations

$$
\frac{d}{d t} \frac{\delta L}{\delta \dot{\mathcal{A}}}=L_{\mathcal{A}} \quad(a) \quad \frac{d}{d t} L_{\dot{q}}=L_{q} \quad \text { (b) }
$$

for the Lagrangian (2.10). Further, the equation (2.15), (a) is equivalent to the Maxwell equations (2.3) with the constraints (2.4), and the equation (2.15), (b) is equivalent to the Lorentz force equation (2.5).

Note that the equations (2.14), (a), (b) are equivalent to standard Euler-Lagrange equations (2.15) because the variables $\mathcal{A}, \dot{\mathcal{A}}, q$, and $\dot{q}$ vary in the corresponding linear spaces. So, we will call these variables the "Lagrange variables".

On the other hand, $R \in S O(3)$, and respectively, the variational equation (2.14) (c) cannot be transformed to a Euler-Lagrange equation since $S O(3)$ is not a linear space. We have shown in 3 , Theorem 2.1] that (2.14) (c) is equivalent to the Lorentz torque equation (2.6) using the variational Poincaré equations with the Lagrangian $L$ expressed in suitable coordinates on $T S O(3)$.

In detail, consider an orthonormal basis $\left\{e_{k}\right\}$ with the right orientation in $\mathbb{R}^{3}$. Then

$$
e_{1} \wedge e_{2}=e_{3}, e_{2} \wedge e_{3}=e_{1}, e_{3} \wedge e_{1}=e_{2} .
$$

Let us express the angular velocity in $\left\{e_{k}\right\}: \omega(t)=\sum \omega_{k}(t) e_{k}$. The algebra so(3) of skewsymmetric $3 \times 3$ matrices with the matrix commutator is isomorphic to the algebra $\mathbb{R}^{3}$ with the vector product, through the isomorphism $\mathcal{J}$ of (2.2):

$$
\left(\begin{array}{ccc}
0 & -\omega_{3} & \omega_{2} \\
\omega_{3} & 0 & -\omega_{1} \\
-\omega_{2} & \omega_{1} & 0
\end{array}\right)=\mathcal{J}\left(\omega_{1}, \omega_{2}, \omega_{3}\right) .
$$

Namely, let $A, B \in s o(3), a, b \in \mathbb{R}^{3}$, and $A=\mathcal{J} a, B=\mathcal{J} b$. Then

$$
A B-B A=\mathcal{J}(a \wedge b) .
$$

Further, $\dot{R} R^{-1} \in T_{E} S O(3)$ is the tangent vector $\dot{R}$ of $S O(3)$ at the point $R$ translated to the unit $E$ of $S O(3)$ by the right translation $R^{-1}$. By the linear isomorphism (2.17),

$$
\dot{R} R^{-1}=\sum \omega_{k} \tilde{e}_{k}, \quad \tilde{e}_{k}:=\mathcal{J} e_{k} .
$$

Then

$$
\dot{R}=\dot{R} R^{-1} R=\sum \omega_{k} v_{k}(R), \quad v_{k}(R):=\tilde{e}_{k} R .
$$


As the result, $\dot{R}$ has the same coordinates w.r.t. the vector fields $v_{k}$ at the point $R$ as $\omega$ in the basis $\left\{e_{k}\right\}$. The fields $v_{k}(R)$ are right translations of $\tilde{e}_{k}$ and hence are right-invariant.

In [3, Lemma 6.1] it is shown that for the vector fields $v_{k}$ on $S O(3)$ the following commutation relations hold:

$$
\left[v_{1}, v_{2}\right]=-v_{3}, \quad\left[v_{2}, v_{3}\right]=-v_{1}, \quad\left[v_{3}, v_{1}\right]=-v_{2} .
$$

We will identify vector fields with the corresponding opertors of differentiation. According to the Poincaré theory [2, 6], the equation (2.14) (c) is equivalent to the Poincaré equations

$$
\frac{d}{d t} \hat{L}_{\omega_{k}}(Y(t))=\sum_{i j} c_{i k}^{j} \omega_{i}(t) \hat{L}_{\omega_{j}}(Y(t))+v_{k} \hat{L}(Y(t)), \quad k=1,2,3,
$$

where $Y(t):=(\mathcal{A}(t), q(t), \dot{\mathcal{A}}(t), \dot{q}(t), \omega(t))$ and $\hat{L}(\mathcal{A}, q, \dot{\mathcal{A}}, \dot{q}, \omega)$ is defined as the right hand side of (2.10), and the constants $c_{i k}^{j}$ arise from commutation relations

$$
\left[v_{i}, v_{k}\right](R)=\sum c_{i k}^{j} v_{j}(R)
$$

In Appendix A, we recall the calculation of the Poincaré equations (2.22). These calculations will be used througough the paper.

Note that the Lagrangian $\hat{L}$ does not depend explicitly on $R$, and hence $v_{k}(\hat{L})=0, k=1,2,3$. Then the corresponding Poincaré equations read

$$
\frac{d}{d t} \frac{\partial \hat{L}(\omega(t))}{\partial \omega_{k}}=\sum_{i j} c_{i k}^{j} \omega_{i} \frac{\partial \hat{L}(\omega(t))}{\partial \omega_{j}}, \quad k=1,2,3 .
$$

In our case (2.21) and (A.6) imply

$$
c_{21}^{3}=c_{32}^{1}=c_{13}^{2}=1, \quad c_{31}^{2}=c_{12}^{3}=c_{23}^{1}=-1, \text { all the rest } c_{i k}^{j}=0 .
$$

Thus, we can rewrite $(\underline{2.23})$ as

$$
\frac{d}{d t} \frac{\partial \hat{L}(\omega(t))}{\partial \omega}=\omega \wedge \frac{\partial \hat{L}(\omega(t))}{\partial \omega}
$$

where $\frac{\partial \hat{L}}{\partial \omega}$ is the column vector with the components $\frac{\partial \hat{L}}{\partial \omega_{k}}, k=1,2,3$.

We summarize the situation as follows, see [3]. The Lagrangian $\hat{L}$ depends on two groups of variables: on the "Lagrangian variables" $\mathcal{A}, \dot{\mathcal{A}}, q, \dot{q}$ and on the variables $\omega_{k}$ which we will call the "Poincaré variables". The variational equations (2.14) (a), (b) imply the Maxwell-Lorentz equations (2.3) $-(2.5)$, while (2.14) (c) give the Lorentz torque equations (2.6) .

\section{Invariants for the Poincaré equations}

When the external fields possess a symmetry with respect to the Lagrangian variables, the corresponding conservation laws are given by the Noether theorem on invariants [1]. In this section we extend the Noether theory to the Poincaré equations.

Let $v_{1}(g), \ldots, v_{n}(g)$ be vector fields on an $n$-dimensional manifold $M$ which are linearly independent at each point $g \in M$. In particular such vector fields exist for any open region $M \subset \mathbb{R}^{n}$. Then $T M$ is isomorphic to $M \times \mathbb{R}^{n}$, and any function $L(g, \dot{g})$ on $T M$ can be expressed in the Poincaré variables $g, \omega$ :

$$
\hat{L}(g, \omega):=L(g, \dot{g}), \quad \dot{g}=\sum \omega_{k} v_{k}(g)
$$


In [6], Poincaré discovered that the corresponding Hamilton least action principle is equivalent to the equations

$$
\frac{d}{d t} \hat{L}_{\omega_{k}}(g(t), \omega(t))=\sum_{i j} c_{i k}^{j}(g) \omega_{i} \hat{L}_{\omega_{j}}(g, \omega)+v_{k}(g) \hat{L}(g, \omega), \quad k=1, \ldots, n .
$$

where the "structure constants" $c_{i k}^{j}(g)$ arise from commutation relations

$$
\left[v_{i}, v_{j}\right](g)=\sum c_{i j}^{k}(g) v_{k}(g), \quad g \in M
$$

see details in Appendix A. Obviously, (2.22) is the particular case of (3.2).

Here we develop the corresponding theory of invariant for the Poincaré equations. Let us start with the energy conservation.

Theorem 3.1 The "energy"

$$
E:=\hat{L}_{\omega} \cdot \omega-\hat{L}=\sum_{k} \hat{L}_{\omega_{k}} \omega_{k}-\hat{L}
$$

is conserved along the paths of the Poincaré equations (3.2).

Proof Let a smooth path $(g(t), \omega(t))$ satisfy Poincaré equations (3.2). Let us compute

$$
\begin{aligned}
\frac{d}{d t}\left(\hat{L}_{\omega} \cdot \omega-\hat{L}\right) & =\frac{d}{d t} \hat{L}_{\omega} \cdot \omega+\hat{L}_{\omega} \cdot \dot{\omega}-\hat{L}_{g} \cdot \dot{g}-\hat{L}_{\omega} \cdot \dot{\omega}=\sum_{k} \frac{d}{d t} \hat{L}_{\omega_{k}} \omega_{k}-\hat{L}_{g} \cdot \dot{g} \\
& =\sum_{k}\left(\sum_{i j} c_{i k}^{j} \omega_{i} \hat{L}_{\omega_{j}}+v_{k}(\hat{L})\right) \omega_{k}-\hat{L}_{g} \cdot \dot{g}
\end{aligned}
$$

by (3.2). Note that $\hat{L}_{g} \cdot \dot{g}=\hat{L}_{g} \cdot \sum \omega_{k} v_{k}=\sum \omega_{k} \hat{L}_{g} \cdot v_{k}=\sum v_{k}(\hat{L}) \omega_{k}$. Thus, we obtain

$$
\frac{d}{d t}\left(\hat{L}_{\omega} \cdot \omega-\hat{L}\right)=\sum_{k}\left(\sum_{i j} c_{i k}^{j} \omega_{i} \hat{L}_{\omega_{j}}\right) \omega_{k}=\sum_{j} \hat{L}_{\omega_{j}} \sum_{i k} c_{i k}^{j} \omega_{i} \omega_{k}=0
$$

since $\sum_{i k} c_{i k}^{j} \omega_{i} \omega_{k}=0$ by skew-symmetry property (A.1) of the coefficients $c_{i k}^{j}$.

Remark 3.2 In the Lagrangian case (i.e., when $M$ is a linear space and $\omega=\dot{g}$ ), the invariant (3.3) coincides with the standard energy functional.

Now let us consider general case of a one-parametric group of diffeomorphisms $h^{s}: M \rightarrow M$ (in particular, $h^{0}=I d_{M}$ ).

Definition 3.3 The Poincaré invariant I and the corresponding "current" $w=\left(w_{1}, \ldots, w_{n}\right)$ are defined as

$$
I(g, \omega):=\sum \hat{L}_{\omega_{k}} w_{k}(g),\left.\quad \frac{d h^{s} g}{d s}\right|_{s=0}=\sum w_{k}(g) v_{k}(g)
$$

These definitions generalize the corresponding Noether formulas [1] to the case of Poincaré equations.

Let the Lagrangian $L$ be invariant with respect to the diffeomorphisms $h^{s}$, i.e.

$$
L\left(h^{s} g, d h^{s} \dot{g}\right)=L(g, \dot{g}), \quad(g, \dot{g}) \in T M, \quad s \in \mathbb{R} .
$$


Theorem 3.4 Let condition (3.7) hold. Then the function $I(g, \omega)$ is conserved along the paths of the Poincaré equations (3.2).

Proof Let a smooth path $(g(t), \omega(t))$ satisfy Poincaré equations (3.2). Let us denote $g(s, t):=$ $h^{s} g(t)$ and write

$$
\dot{g}(s, t)=d h^{s} \dot{g}(t)=\sum \omega_{k}(s, t) v_{k}(g(s, t)) .
$$

In particular, $g(0, t)=g(t)$ and $\dot{g}(t):=\sum \omega_{k}(t) v_{k}(g(t))$. By (3.7), the quantity

$$
\hat{L}(g(s, t), \omega(s, t)):=L\left(g(s, t), \sum_{k} \omega_{k}(s, t) v_{k}(g(s, t))\right)=L\left(g(s, t), d h^{s} \dot{g}(t)\right)
$$

does not depend on $s$; here $\omega(s, t)=\left(\omega_{1}(s, t), \ldots, \omega_{n}(s, t)\right)$. Denote by prime the derivative in $s$, and by dot the derivative in $t$. Then we obtain

$$
0=\frac{d}{d s} \hat{L}(g(s, t), \omega(s, t))=\hat{L}_{g} \cdot g^{\prime}+\sum_{k} \hat{L}_{\omega_{k}} \omega_{k}^{\prime}=\hat{L}_{g} \cdot g^{\prime}+\sum_{k} \hat{L}_{\omega_{k}}\left(\sum_{i j} c_{i j}^{k} \omega_{i} w_{j}+\dot{w}_{k}\right)=: S
$$

by the formula (A.2) of Appendix A. First we change the order of summation on the right-hand side:

$$
S=\hat{L}_{g} \cdot g^{\prime}+\sum_{k} \hat{L}_{\omega_{k}} \dot{w}_{k}+\sum_{j}\left(\sum_{i k} c_{i j}^{k} \omega_{i} \hat{L}_{\omega_{k}}\right) w_{j}
$$

Next we wish to evaluate the term $\sum_{i k} c_{i j}^{k} \omega_{i} \hat{L}_{\omega_{k}}$ for $s=0$. Namely, $g(0, t)=g(t)$ together with $\omega(0, t)=\omega(t)$ satisfy the Poincaré equations (3.2) . Hence, for $s=0$

$$
\begin{aligned}
S & =\hat{L}_{g} \cdot g^{\prime}+\sum_{k} \hat{L}_{\omega_{k}} \dot{w}_{k}+\sum_{j}\left(\frac{d}{d t} \hat{L}_{\omega_{j}}-v_{j}(\hat{L})\right) w_{j} \\
& =\sum_{k} \hat{L}_{\omega_{k}} \dot{w}_{k}+\sum_{j} \frac{d}{d t} \hat{L}_{\omega_{j}} \cdot w_{j}+\hat{L}_{g} \cdot g^{\prime}-\sum_{j} v_{j}(\hat{L}) w_{j} .
\end{aligned}
$$

However, the definition of the current $w$ in (3.6) implies that

$$
\hat{L}_{g} \cdot g^{\prime}-\sum_{j} v_{j}(\hat{L}) w_{j}=\sum_{j} \hat{L}_{g} \cdot v_{j} w_{j}-\sum_{j} v_{j}(\hat{L}) w_{j}=0
$$

Therefore, (3.10) gives

$$
S=\sum_{k} \hat{L}_{\omega_{k}} \dot{w}_{k}+\sum_{k} \frac{d}{d t} \hat{L}_{\omega_{k}} \cdot w_{k}=\frac{d}{d t}\left(\sum_{k} \hat{L}_{\omega_{k}} w_{k}\right)=\dot{I}(t)
$$

The proof is complete, since $S=0$ by $(3.8)$.

Remark 3.5 Let $M=\mathbb{R}^{n}$ and the vector fields $v_{k}=\nabla_{g_{k}}$ be the commuting fields of differentiations w.r.t. coordinates $g_{k}$. Then Poincar'e equations (3.2) read as the Euler-Lagrange equations, and the Poincaré invariant (3.6) coinsides with the Noether invariant $\left.L_{\dot{g}} \cdot \frac{d h^{s} g}{d s}\right|_{s=0}$. 


\section{Invariants for the Lagrange-Poincaré equations}

Here we generalize the theory of the previous section to systems with the configuration space $Y \times M$, where $Y$ is a Hilbert space either of finite or infinite dimension, while $M$ is a finitedimensional manifold endowed with the vector fields $v_{k}(g)$ as above. Then $T Y \simeq Y \times Y$ and $T M \simeq M \times \mathbb{R}^{n}$.

Let $L(X, V, g, \dot{g})$ be a differentiable Lagrangian which is defined on $T Y \times T M$. Let us define

$$
\hat{L}(X, V, g, \omega):=L(X, V, g, \dot{g}), \quad \dot{g}=\sum \omega_{k} v_{k}(g) .
$$

Let a smooth path $(X(t), V(t)), g(t), \omega(t)$ satisfy standard Euler-Lagrange equations w.r.t. the variables $(X, V)$ and Poincaré equations w.r.t. the variables $(g, \omega)$ :

$$
\left\{\begin{aligned}
\frac{d}{d t} \hat{L}_{V} & =L_{X} \\
\frac{d}{d t} \hat{L}_{\omega_{k}} & =\sum_{i j} c_{i k}^{j}(g) \omega_{i} \hat{L}_{\omega_{j}}+v_{k}(g) \hat{L}, k=1, \ldots, n .
\end{aligned}\right.
$$

Theorem 4.1 Let (4.2) hold. Then the energy

$$
E:=\hat{L}_{V} \cdot V+\hat{L}_{\omega} \cdot \omega-\hat{L}
$$

is conserved along the path.

Proof Differentiating formally, we get

$$
\begin{gathered}
\frac{d}{d t}\left(\hat{L}_{V} \cdot V+\hat{L}_{\omega} \cdot \omega-\hat{L}\right)= \\
=\left(\frac{d}{d t} \hat{L}_{V}(X, V) \cdot V-\hat{L}_{X} \cdot \dot{X}\right)+\left(\frac{d}{d t} \hat{L}_{\omega}(g, \omega) \cdot \omega-\hat{L}_{g} \cdot \dot{g}\right)=0 .
\end{gathered}
$$

Indeed, here the first bracket of the last line vanishes by the first equation of (4.2). The second bracket vanishes by the second equation of (4.2) that follows by the calculations (3.4) $-(3.5)$.

Further, consider a one-parametric group of diffeomorphisms

$$
h^{s}:(X, g) \mapsto\left(h_{1}^{s}(X), h_{2}^{s}(g)\right) .
$$

Let us suppose that the Lagrangian functional is $h^{s}$-invariant, i.e.

$$
L\left(h_{1}^{s} X, d h_{1}^{s} V, h_{2}^{s} g, d h_{2}^{s} \dot{g}\right) \equiv L(X, V, g, \dot{g}) .
$$

Theorem 4.2 Let (4.2), (4.5) hold. Then the sum

$$
\left.\hat{L}_{V} \cdot \frac{d h_{1}^{s} X}{d s}\right|_{s=0}+\sum \hat{L}_{\omega_{k}} w_{k}(g)
$$

is conserved along the path.

Proof Let $X(s, t):=h_{1}^{s} X, g(s, t):=h_{2}^{s} g$, and let $\omega(s, t)$ be defined as above. Then $\dot{g}(s, t)=$ $\sum_{k} v_{k}(g(s, t)) \omega_{k}(s, t)$, and formally,

$$
0=\frac{d}{d s} \hat{L}(X(s, t), \dot{X}(s, t), g(s, t), \omega(s, t))=\hat{L}_{X} \cdot X^{\prime}+\hat{L}_{\dot{X}} \cdot \dot{X}(s, t)^{\prime}+\hat{L}_{g} \cdot g^{\prime}+\sum \hat{L}_{\omega_{k}} \omega_{k}^{\prime} .
$$

At $s=0$, the sum of the first two terms reduces to $\frac{d}{d t}\left(\left.L_{\dot{X}} \cdot \frac{d h_{1}^{s} X}{d s}\right|_{s=0}\right)$ like in the proof of the standard theorem on Noether invariants [1. The sum of the last two terms transforms to $\frac{d}{d t}\left(\sum \hat{L}_{\omega_{k}} w_{k}(g)\right)$ like in the proof of Theorem 3.4 (calculations $\left.(3.8)-(3.11)\right)$. 


\section{Conservation laws for Maxwell-Lorentz equations}

We now apply the theory of Noether invariants and Poincare invariants for our system of MaxwellLorentz equations with rotating charge. As above, we denote

$$
\hat{L}(\mathcal{A}, q, \dot{\mathcal{A}}, \dot{q}, \omega)=L(\mathcal{A}, q, R, \dot{\mathcal{A}}, \dot{q}, \dot{R})
$$

where $\omega=\left(\omega_{1}, \omega_{2}, \omega_{3}\right)$ is defined by (2.20), i.e., $\omega_{k}$ are coordinates of $\dot{R}$ in the basis $v_{1}(R), v_{2}(R)$, $v_{3}(R)$; recall that $\hat{L}$ does not depend explicitly on $R$.

\section{$5.1 \quad$ Energy}

Let us note that $L$ does not depend on $\dot{A}_{0}$. By Theorem 4.1, we come formally to the following statement:

Corollary 5.1 Suppose $A_{0}^{\text {ext }}$ end $A^{\text {ext }}$ do not depend on time. Then the functional

$$
E(\mathcal{A}, q, \dot{\mathcal{A}}, \dot{q}, R, \omega):=\hat{L}_{\dot{A}} \cdot \dot{A}+\hat{L}_{\dot{q}} \cdot \dot{q}+\hat{L}_{\omega} \cdot \omega-\hat{L}
$$

is conserved along the regular solutions of the Maxwell-Lorentz system (2.3)-(2.5).

\subsection{Momentum}

Let the external field

$$
\mathcal{A}^{e x t}(x)=\left(A_{0}^{e x t}(x), A^{e x t}(x)\right) \text { do not depend on } x_{k} \text { for some } k \text {. }
$$

Then the Lagrangian (5.1) is invariant w.r.t to the one-parametric group of spatial translations

$$
h_{k}^{s}(\mathcal{A}(x), q)=\left(\mathcal{A}\left(x-s e_{k}\right), q+s e_{k}\right),
$$

where $e_{k} \in \mathbb{R}^{3}$ is the corresponding basis vector. Since the group acts only on the Lagrange coordinates $X:=(\mathcal{A}, q), V:=(\dot{\mathcal{A}}, \dot{q})$, we may formally apply the Noether theory [1, 5] and obtain

Corollary 5.2 Under the condition (5.3) the functional

$$
P_{k}=P_{k}(X, V, R, \omega):=\left.\hat{L}_{V} \cdot \frac{d h_{k}^{s} X}{d s}\right|_{s=0}
$$

is conserved for regular solutions to the Maxwell-Lorentz system (2.3)-(2.5).

Definition $5.3 P_{k}$ is called $k$-th component of momentum of the state $(X, V, R, \omega)$.

\subsection{Angular momentum}

Let the external potential $\mathcal{A}^{\text {ext }}$ be axially symmetric,

$$
A_{0}^{e x t}\left(U_{k} x\right)=A_{0}^{e x t}(x), \quad A^{e x t}\left(U_{k} x\right)=U_{k} A^{e x t}(x),
$$

where $U_{k}$ is any rotation around the axis $O x_{k}$. 
Lemma 5.4 Let (5.6) hold. Then the Lagrangian (2.10) is invariant w.r.t. the axial rotations

$$
\begin{aligned}
A_{0}(x) \mapsto A_{0}\left(U_{k}^{-1} x\right), & A(x) \mapsto U_{k} A\left(U_{k}^{-1} x\right), \quad \dot{A}(x) \mapsto U_{k} \dot{A}\left(U_{k}^{-1} x\right), \\
R & \mapsto U_{k} R, \quad \dot{R} \mapsto U_{k} \dot{R}, \\
q & \mapsto U_{k} q, \quad \dot{q} \mapsto U_{k} \dot{q} .
\end{aligned}
$$

Proof By (2.8) the transforms (5.7) of the potentials induce the following transforms of the fields:

$$
E(x) \mapsto U_{k} E\left(U_{k}^{-1} x\right), \quad B(x) \mapsto U_{k} B\left(U_{k}^{-1} x\right)
$$

Further, we have, in operator notations, $\mathcal{J} \omega=\omega \wedge$, where $\omega \wedge$ is the operator of the vector product by $\omega$ in $\mathbb{R}^{3}$. Then it is easy to check that $\mathcal{J}\left(U_{k} \omega\right)=U_{k} \mathcal{J}(\omega) U_{k}^{-1}$. Thus, for $\omega=\mathcal{J}^{-1} \dot{R} R^{-1}$ we obtain $\mathcal{J} \omega=\dot{R} R^{-1}$ and hence $\mathcal{J}\left(U_{k} \omega\right)=U_{k}\left(\dot{R} R^{-1}\right) U_{k}^{-1}=\left(U_{k} \dot{R}\right)\left(U_{k} R\right)^{-1}$. Finally,

$$
U_{k} \omega=\mathcal{J}^{-1}\left(U_{k} \dot{R}\right)\left(U_{k} R\right)^{-1} \text {. }
$$

This means that the transforms (5.8) induce the following transform of $\omega$ :

$$
\omega \mapsto U_{k} \omega
$$

Now it is easy to check, in view of axial symmetry of $\mathcal{A}^{\text {ext }}$, the invariance of $L$ w.r.t. the transforms (5.10), (5.9), (5.11), since $\rho$ is spherically symmetric.

Recall that $\tilde{e}_{k}$ is the image of the basis vector $e_{k}$ w.r.t. the isomorphism (2.17). By Lemma 5.4 the Lagrangian $\hat{L}$ (5.1) is invariant w.r.t. the spatial rotations (5.9), (5.10), (5.11). In particular, $\hat{L}$ is invariant under the transform group $h_{k}^{s}=e^{s \tilde{e}_{k}} \in S O(3)$.

In detail, we have the situation of previous section when $\hat{L}$ depends on Lagrangian variables $(X ; V)=(\mathcal{A}, q ; \dot{\mathcal{A}}, \dot{q})$ and on Poincaré variables $(R, \omega)$. The action of this group on the state $(X, R)$ reads

$$
h_{k}^{s}(X, R)=\left(\alpha_{k}^{s} X, \beta_{k}^{s} R\right): \quad \alpha_{k}^{s} X=\left(A_{0}\left(e^{-s \tilde{e}_{k}} x\right), e^{s \tilde{e}_{k}} A\left(e^{-s \tilde{e}_{k}} x\right), e^{s \tilde{e}_{k}} q\right) ; \quad \beta_{k}^{s} R=e^{s \tilde{e}_{k}} R .
$$

The currents $w_{1}^{k}(R), w_{2}^{k}(R), w_{3}^{k}(R)$ are defined from

$$
\left.\frac{d \beta_{k}^{s} R}{d s}\right|_{s=0}=\sum_{j=1}^{3} w_{j}^{k}(R) v_{j}(R), \quad R \in S O(3) .
$$

Hence, by Theorem 4.2 we come to the following statement:

Corollary 5.5 Under the condition (5.6) the quantity

$$
M_{k}=M_{k}(X, V, R, \omega):=\left.\hat{L}_{V} \cdot \frac{d \alpha_{k}^{s} X}{d s}\right|_{s=0}+\sum_{j=1}^{3} \hat{L}_{\omega_{j}} w_{j}^{k}(R)
$$

is conserved for regular solutions to the Maxwell-Lorentz system (2.3)-(2.5).

Definition 5.6 $M_{k}$ is called $k$-th component of angular momentum of the state $(X, V, R, \omega)$. 


\section{Expressions for energy and momenta}

Let us show that the Poincaré invariants from previous section coincide with classical known expressions considered in [4] (where their conservation was shown by direct calculation).

Proposition 6.1 The invariants for the Maxwell-Lorentz system (2.3)-(2.5) read as follows:

i) The energy reads

$$
E=\frac{1}{2} \int\left(|E(x)|^{2}+|B(x)|^{2}\right) d x+\frac{1}{2} \dot{q}^{2}+\frac{1}{2} I \omega^{2}+\int A_{0}^{e x t}(x) \rho(x-q) d x .
$$

ii) The momentum reads

$$
P=\dot{q}+\int E(x) \wedge B(x) d x+\int A^{e x t}(x) \rho(x-q) d x .
$$

iii) The angular momentum reads

$$
M=q \wedge \dot{q}+I \omega+\int x \wedge E(x) \wedge B(x) d x+\int x \wedge A^{e x t}(x) \rho(x-q) d x .
$$

Proof i) By (5.1) and (2.10), one has

$$
\hat{L}_{\dot{A}} \cdot \dot{A}=-\int E \cdot \dot{A} d x, \hat{L}_{\dot{q}} \cdot \dot{q}=\dot{q}^{2}+\int \dot{q} \cdot\left(A+A^{e x t}\right) \rho(x-q) d x,
$$

and

$$
\hat{L}_{\omega} \cdot \omega=I \omega^{2}+\int(\omega \wedge(x-q)) \cdot\left(A+A^{e x t}\right) \rho(x-q) d x
$$

Then

$$
\begin{aligned}
E= & \hat{L}_{\dot{A}} \cdot \dot{A}+\hat{L}_{\dot{q}} \cdot \dot{q}+\hat{L}_{\omega} \cdot \omega-\hat{L}=\frac{1}{2} \dot{q}^{2}+\frac{1}{2} I \omega^{2}+\frac{1}{2} \int\left(|B|^{2}-|E|^{2}\right) d x \\
& +\int\left(-E \cdot \dot{A}+A_{0} \rho(x-q) d x+\int A_{0}^{e x t} \rho(x-q)\right) d x .
\end{aligned}
$$

Since

$$
\begin{aligned}
\int\left(-E \cdot \dot{A}+A_{0} \rho(x-q)\right) d x & =\int\left(-E \cdot \dot{A}+A_{0} \cdot \nabla E\right) d x \\
& =-\int E\left(\dot{A}-\nabla A_{0}\right) d x=\int E^{2} d x,
\end{aligned}
$$

formula (6.4) reads (6.1).

ii) Let us compute $P_{j}$. Formula (5.4) implies

$$
\left.\frac{d h_{j}^{s}(X)}{d s}\right|_{s=0}=-\left(e_{j} \cdot \nabla A(x), e_{j}\right) .
$$

Then

$$
\begin{aligned}
P_{j} & =\left.L_{V} \cdot \frac{d h_{j}^{s}(X)}{d s}\right|_{s=0}=-L_{\dot{A}} \cdot\left(e_{j} \cdot \nabla\right) A+L_{\dot{q}} \cdot e_{j} \\
& =-\int\left(\nabla A_{0}+\dot{A}\right) \cdot\left(e_{j} \cdot \nabla\right) A d x+\dot{q} \cdot e_{j}+\int e_{j} \cdot A \rho(x-q) d x+\int A_{j}^{e x t} \rho(x-q) d x \\
& =\dot{q}_{j}+\int A_{j} \rho(x-q) d x-\int\left(\nabla A_{0}+\dot{A}\right) \cdot \partial_{j} A d x+\int A_{j}^{e x t} \rho(x-q) d x
\end{aligned}
$$


By partial integration

$$
\begin{aligned}
\int A_{j}(x) \rho(x-q) d x & =\int A_{j}(\nabla \cdot E) d x=\int A_{j} \nabla \cdot\left(-\nabla A_{0}-\dot{A}\right) d x \\
& =\int A_{j}\left(-\Delta A_{0}-\nabla \dot{A}\right) d x=\int\left(\nabla A_{0} \cdot \nabla A_{j}+(\dot{A} \cdot \nabla) A_{j}\right) d x
\end{aligned}
$$

Hence,

$$
P_{j}=\dot{q}_{j}+\int\left(\nabla A_{0} \cdot \nabla A_{j}+(\dot{A} \cdot \nabla) A_{j}\right) d x-\int\left(\nabla A_{0} \cdot \partial_{j} A+\dot{A} \cdot \partial_{j} A\right) d x+\int A_{j}^{e x t} \rho(x-q) d x .
$$

On the other hand, the j-th component of the RHS of (6.2) equals

$$
\dot{q}_{j}+\int(E \wedge B)_{j} d x+\int A_{j}^{e x t} \rho(x-q) d x .
$$

Insert $E=-\dot{A}-\nabla A_{0}, B=\nabla \wedge A$ and obtain

$$
\dot{q}_{j}+\int A_{j}^{e x t} \rho(x-q) d x+\int\left((\dot{A} \cdot \nabla) A_{j}-\dot{A} \cdot \partial_{j} A+\nabla A_{0} \cdot \nabla A_{j}-\nabla A_{0} \cdot \partial_{j} A\right) d x
$$

which coincides with (6.7).

iii) For concreteness let us compute $M_{1}$. Then

$$
\alpha_{1}^{s}(X)=\left(A_{0}\left(e^{-s \tilde{e}_{1}} x\right), e^{s \tilde{e}_{1}} A\left(e^{-s \tilde{e}_{1}} x\right), e^{s \tilde{e}_{1}} q\right) .
$$

One has

$$
\begin{gathered}
\left.\frac{d \alpha_{1}^{s} X}{d s}\right|_{s=0}=\left(-\tilde{e}_{1} e^{-s \tilde{e}_{1}} x \cdot \nabla\right) \\
\left.A_{0}\left(e^{-s \tilde{e}_{1}} x\right), \tilde{e}_{1} e^{s \tilde{e}_{1}} A\left(e^{-s \tilde{e}_{1}} x\right)+e^{s \tilde{e}_{1}}\left(-\tilde{e}_{1} e^{-s \tilde{e}_{1}} x \cdot \nabla\right) A\left(e^{-s \tilde{e}_{1}} x\right), \tilde{e}_{1} e^{s \tilde{e}_{1}} q\right)\left.\right|_{s=0} \\
=\left(\tilde{e}_{1} A_{0}(x), \tilde{e}_{1} A(x)-\left(\tilde{e}_{1} x \cdot \nabla\right) A(x), \tilde{e}_{1} q\right)
\end{gathered}
$$

Further,

$$
\left.\frac{d \beta_{1}^{s} R}{d s}\right|_{s=0}=\left.\frac{d e^{s \tilde{e}_{1}} R}{d s}\right|_{s=0}=\tilde{e}_{1} R=v_{1}(R)
$$

by definition (2.20) of the fields $v_{k}(R)$. Hence, for the currents $w_{j}^{1}$ of (5.12) we have $w_{1}^{1}=1$, $w_{2}^{1}=w_{3}^{1}=0$. Then, since $\hat{L}$ does not depend on $\dot{A}_{0}$,

$$
\begin{aligned}
M_{1}= & \hat{L}_{\dot{A}} \cdot\left(\tilde{e}_{1} A(x)-\left(\tilde{e}_{1} x \cdot \nabla\right) A(x)\right)+\hat{L}_{\dot{q}} \cdot\left(\tilde{e}_{1} q\right)+\hat{L}_{\omega_{1}} \\
= & \int\left(\dot{A} \cdot\left(\tilde{e}_{1} A(x)-\left(\tilde{e}_{1} x \cdot \nabla\right) A(x)\right)+\nabla A_{0} \cdot\left(\tilde{e}_{1} A(x)-\left(\tilde{e}_{1} x \cdot \nabla\right) A(x)\right)\right) d x \\
& +\dot{q} \cdot\left(\tilde{e}_{1} q\right)+\int\left(\tilde{e}_{1} q\right) \cdot\left(A+A^{e x t}\right) \rho(x-q) d x+I \omega \cdot e_{1}+\int\left(e_{1} \wedge(x-q)\right) \cdot\left[A+A^{e x t}\right] \rho(x-q) d x \\
= & (q \wedge \dot{q})_{1}+I \omega_{1}+\int\left(x_{2} A_{3}^{e x t}-x_{3} A_{2}^{e x t}\right) \rho(x-q) d x \\
& +\int\left(x_{2} A_{3}-x_{3} A_{2}\right) \rho(x-q) d x+\int\left(\dot{A}+\nabla A_{0}\right) \cdot\left(\left(0,-A_{3}, A_{2}\right)+\left(x_{3} \partial_{2}-x_{2} \partial_{3}\right) A\right) d x
\end{aligned}
$$


We have to prove that this expression equals to the first component of the RHS of (6.3). It

suffices to prove that the last line (6.8) equals to the first component of $\int x \wedge(E \wedge B) d x$. Indeed, $\rho(x-q)=\nabla \cdot E=\nabla \cdot\left(-\nabla A_{0}-\dot{A}\right)$, hence

$$
\int\left(x_{2} A_{3}-x_{3} A_{2}\right) \rho(x-q) d x=\int\left(x_{2} A_{3}-x_{3} A_{2}\right)\left(-\nabla \dot{A}-\nabla^{2} A_{0}\right) d x=\int \nabla\left(x_{2} A_{3}-x_{3} A_{2}\right)\left(\dot{A}+\nabla A_{0}\right) d x .
$$

Then the line (6.8) transforms to

$$
\begin{aligned}
& \int\left(\partial_{1}\left(x_{2} A_{3}-x_{3} A_{2}\right)\left(\dot{A}_{1}+\partial_{1} A_{0}\right)+x_{2} \partial_{2} A_{3}\left(\dot{A}_{2}+\partial_{2} A_{0}\right)-x_{3} \partial_{3} A_{2}\left(\dot{A}_{3}+\partial_{3} A_{0}\right)\right) d x \\
+ & \int\left(\left(x_{3} \partial_{2}-x_{2} \partial_{3}\right) A_{1}\left(\dot{A}_{1}+\partial_{1} A_{0}\right)-x_{2} \partial_{3} A_{2}\left(\dot{A}_{2}+\partial_{2} A_{0}\right)+x_{3} \partial_{2} A_{3}\left(\dot{A}_{3}+\partial_{3} A_{0}\right)\right) d x .
\end{aligned}
$$

On the other hand, substitute $E=-\dot{A}-\nabla A_{0}, B=\nabla \wedge A$ and obtain that the first component of $\int x \wedge(E \wedge B) d x$ equals

$$
\begin{array}{r}
\int x_{2}\left(\left(\partial_{1} A_{3}-\partial_{3} A_{1}\right)\left(\dot{A}_{1}+\partial_{1} A_{0}\right)+\left(\partial_{2} A_{3}-\partial_{3} A_{2}\right)\left(\dot{A}_{2}+\partial_{2} A_{0}\right)\right) d x \\
-\int x_{3}\left(\left(\partial_{3} A_{2}-\partial_{2} A_{3}\right)\left(\dot{A}_{3}+\partial_{3} A_{0}\right)+\left(\partial_{1} A_{2}-\partial_{2} A_{1}\right)\left(\dot{A}_{1}+\partial_{1} A_{0}\right)\right) d x
\end{array}
$$

which coincides with (6.10). The proof is complete.

\section{A Poincaré equations}

Poincaré suggested the form of the Hamilton least action principle for Lagrangian systems on manifolds [6]. We present the derivation of the Poincaré equations [2] since we use some of intermediate calculations.

Let $v_{1}, \ldots, v_{n}$ be vector fields on a $n$-dimensional manifold $M$ which are linearly independent at every point $g \in M$. Then the commutation relations hold,

$$
\left[v_{i}, v_{j}\right](g)=\sum c_{i j}^{k}(g) v_{k}(g), \quad g \in M
$$

where the commutator $\left[v_{i}, v_{j}\right]$ is defined by

$$
\left[v_{i}, v_{j}\right](f):=v_{i}\left(v_{j}(f)\right)-v_{j}\left(v_{i}(f)\right)
$$

and $v(f)$ is the derivative of a smooth function $f$ on $M$ w.r.t. the vector field $v$. Note that by the skew-symmetry property of the commutators one has

$$
c_{i j}^{k}(g)=-c_{j i}^{k}(g), \quad \forall k=1, \ldots, n .
$$

If $g(t)$ is a smooth path in $M$ and $f$ is a smooth function on $M$, one has $\dot{g}(t)=\sum \omega_{i}(t) v_{i}(g(t))$ and

$$
\frac{d}{d t} f(g(t))=f^{\prime}(g(t)) \cdot \dot{g}=f^{\prime}(g(t)) \cdot \sum \omega_{i}(t) v_{i}(g(t))=\sum v_{i}(f) \omega_{i}(t) .
$$

Now consider a variation $g(s, t)$ of the path $g(t)$. Then similarly,

$$
\partial_{s} f(g(s, t))=\sum_{j} v_{j}(f) w_{j}(s, t),
$$


where $w_{j}(s, t)$ are coordinates of $\frac{\partial g}{\partial s}(s, t) \in T_{g(s, t)} M$. Hence

$$
\begin{aligned}
& \partial_{s} \partial_{t} f(g(s, t))=\sum_{i} \sum_{j} v_{j}\left(v_{i}(f)\right) w_{j} \omega_{i}+\sum_{i} v_{i}(f) \omega_{i}^{\prime}, \\
& \partial_{t} \partial_{s} f(g(s, t))=\sum_{j} \sum_{i} v_{i}\left(v_{j}(f)\right) w_{j} \omega_{i}+\sum_{j} v_{j}(f) \dot{w}_{j},
\end{aligned}
$$

where the prime respectively the dot stand for the differentiation in $s$ respectively in $t$. However, the differentiations in $t$ and $s$ commute, hence we obtain by subtraction

$$
\sum_{k} v_{k}(f) \omega_{k}^{\prime}=\sum_{k} \sum_{i j} c_{i j}^{k} \omega_{i} w_{j} v_{k}(f)+\sum_{k} v_{k}(f) \dot{w}_{k} .
$$

Since $f$ is an arbitrary smooth function, we come to the relations

$$
\omega_{k}^{\prime}(s, t)=\sum_{i j} c_{i j}^{k} \omega_{i} w_{j}+\dot{w}_{k}
$$

Further, let us consider a Lagrangian function $L(g, \dot{g})$ on $T M$. Then $L(g, \dot{g})$ can be expressed in the variables $\omega: L(g, \dot{g})=\hat{L}(g, \omega)$. Let us compute the variation of the corresponding action functional taking (A.2) into account:

$$
\begin{gathered}
\frac{d}{d s} \int_{t_{1}}^{t_{2}} \hat{L}(g(s, t), \omega(s, t)) d t=\int_{t_{1}}^{t_{2}}\left(\sum_{k} \frac{\partial \hat{L}}{\partial \omega_{k}} \omega_{k}^{\prime}+\nabla_{g} \hat{L} \cdot g^{\prime}\right) d t= \\
\int_{t_{1}}^{t_{2}}\left[\sum_{k} \frac{\partial \hat{L}}{\partial \omega_{k}}\left(\dot{w}_{k}+\sum_{i j} c_{i j}^{k} \omega_{i} w_{j}\right)+\nabla_{g} \hat{L} \cdot \sum_{k} w_{k} v_{k}\right] d t= \\
\left.\sum_{k} \frac{\partial \hat{L}}{\partial \omega_{k}} w_{k}\right|_{t_{1}} ^{t_{2}}+\int_{t_{1}}^{t_{2}} \sum_{k}\left[-\frac{d}{d t} \frac{\partial \hat{L}}{\partial \omega_{k}}+\sum_{i j} c_{i k}^{j} \omega_{i} \frac{\partial \hat{L}}{\partial \omega_{j}}+v_{k}(\hat{L})\right] w_{k} d t .
\end{gathered}
$$

The variation should be zero by the Hamilton least action principle, under the boundary value conditions

$$
g\left(s, t_{1}\right)=g_{1}, \quad g\left(s, t_{2}\right)=g_{2} .
$$

Since $w_{k}\left(t_{1}\right)=w_{k}\left(t_{2}\right)=0$ by (A.3), we obtain the following Poincaré equations:

$$
\frac{d}{d t} \frac{\partial \hat{L}}{\partial \omega_{k}}=\sum_{i j} c_{i k}^{j} \omega_{i} \frac{\partial \hat{L}}{\partial \omega_{j}}+v_{k}(\hat{L}) .
$$

Remarks 1. If $g$ is expressed in a local map as $\left(g_{1}, \ldots, g_{n}\right) \in \mathbb{R}^{n}$, and $v_{k}=\partial_{g_{k}}$, then (A.4) reduce to the standard Euler-Lagrange equations.

2. If a Lagrangian $L$ does not depend on $g$, then $\hat{L}=\hat{L}(\omega)$ and one has

$$
v_{k}(\hat{L})=0 \text {. }
$$

Indeed, $v_{k}(\hat{L})=\nabla_{g} \hat{L} \cdot v_{k}(g)=0$.

3. Suppose $M=G$ is a Lie group, and let $v_{k}, k=1, \ldots, n$ be independent either left-invariant or right-invariant vector fields on $G$. Then $c_{i j}^{k}(g)$ are constant:

$$
c_{i j}^{k}(g) \equiv c_{i j}^{k}, \quad g \in G .
$$

Acknowledgements The research of A. Komech was supported partly by Austrian Science Fund (FWF): P28152-N35. The research of V. Imaykin and A. Komech were supported partly by the grant of RFBR: 16-01-00100. The authors thank Professor M. Kiessling for useful discussions and remarks. 


\section{References}

[1] V. Arnold, Mathematical Methods of Classical Mechanics, Springer, New York, 1978.

[2] V.I. Arnold, V.V. Kozlov, A.I. Neishtadt, Mathematical Aspects of Classical and Celestial Mechanics, Springer, Berlin, 1997.

[3] V. Imaykin, A. Komech, H. Spohn, On the Lagrangian theory for rotating charge in the Maxwell field, Phys Lett A 379 (2015), 5-10.

[4] M. Kiessling, Classical electron theory and conservation laws, Phys Lett A 258 (1999), 197-204

[5] A.I. Komech, Quantum Mechanics: Genesis and Achievements, Springer, Dordrecht, 2013.

[6] H. Poincaré, Sur une forme nouvelle des équations de la mécanique C. R. 132 (1901), 369-371.

[7] H. Spohn, Dynamics of Charged Particles and their Radiation Field, Cambridge University Press, Cambridge, 2004. 